$\mathrm{CH}^{+}, \mathrm{CH}_{2}{ }^{+}, \mathrm{C}_{2} \mathrm{H}_{2}{ }^{+}, \mathrm{CH}_{5}{ }^{+}, \mathrm{H}_{2} \mathrm{O}^{+}$and others, with $\mathrm{N}_{2} \mathrm{O}$ and $\mathrm{NO}$ were studied. The rates are often relatively high, and many of the reactions are clearly likely to be important in interstellar chemistry. The reaction schemes indicate once again the importance of the $\mathrm{HCO}^{+}$molecular ion and of reactions with $\mathrm{H}_{2}$.

This work not only provides much useful data but also demonstrates the importance of laboratory studies in the interpretation of interstellar chemistry and physics.

\title{
Geomagnetic secular variation and the conductivity of the lower mantle
}

\section{from David R, Barraclough}

THE slow changes in the Earth's magnetic field with time provide one of the very few means to investigate the dynamics of the Earth's fluid core and the electrical conductivity of the lower mantle. Observations of the geomagnetic field changes - the secular variation - have been made ever since the seventeenth century because of their practical importance for those using magnetic charts. Nowadays, a network of 200 permanent magnetic observatories provides very accurate data free from spurious effects associated with changes of observing site.

The task of forecasting the secular variation for several years ahead is complicated by any sudden changes which may occur. Such a change appears to have taken place in about 1970. It was first noticed in the data from European observatories (Courtillot et al. Comptes rendus D287, 1095; 1978) but it has since been shown to have had world-wide effects (Ducruix et al., Geophys. J.R. astr. Soc. 61, 73; 1980; Malin et al. Ebro Obs. 75th Anniversary Volume in the press). The phenomenon manifested itself as a sudden change in the secular acceleration, the second time-derivative of the main geomagnetic field (Barraclough \& Malin, Geophys. J.R. astr. Soc. 58, 785; 1979). Ducruix et al. found a positive change in the east component of the secular acceleration over Europe and central Russia and a negative change in this component over eastern Asia and northwestern North America. Malin et al. examined the changes in the secular acceleration of the magnetic declination, the horizontal intensity and the vertical component. Their results for the declination are in agreement with the French results and they also find regional patterns in the other elements. Interestingly, all three elements suffered a sudden decrease in the secular acceleration over North America. Using a homogeneous set of observatory annual means covering the period from 1961 to 1978, Malin et al. have produced a spherical harmonic model of the phenomenon which clearly shows the details of its global distribution. There is general agreement that these sudden changes occurred on a time-scale short in comparison with that of the secular variation itself: certainly less than 4 years.

Most geomagnetic parameters vary with the sunspot cycle, which has a period of approximately 11 years. Observatory annual mean values show such a sunspot cycle variation (RV) which, although small in amplitude, can have a significant effect on secular variation estimates derived from the annual means. The source of RV is believed to lie above the Earth's surface, within the magnetosphere. Because the Earth has a finite electrical conductivity, these external magnetic field variations will induce electric currents within the Earth, together with associated magnetic fields. Thus, what is observed at the Earth's surface is a combination of the external inducing field and the internal induced field. It is possible, however, by considering the horizontal components and the vertical component of the variation field separately, to separate the internal and external parts. The ratio of the amplitudes of the two parts and their phase difference can then be used to investigate the conductivity of the regions of the Earth where the induced currents flow - the lower mantle in the case of RV. Three studies of $R V$ have recently been published. Ducruix et al. (op. cit.) used the data from the 10 European observatories which provided the evidence for the sudden change in secular acceleration. The data spanned the interval 1947 to 1977. Yukutake and Cain (J. Geomagn. Geoelect. Kyoto 31, 509; 1979) based their main conclusions on data from 21 observatories, globally distributed, and covering the period from 1900 to 1973 . Harwood and Malin (Geophys. J.R. astr. Soc. 50, 605, 1977) used all available data from 81 observatories with a world-wide distribution. Their earliest data were for 1842 and the most recent for 1974 . The table below gives the amplitude ratios $(I / E)$ and the phase differences $(1-\varepsilon)$ which result from these studies. The estimated uncertainties are approximately 0.1 for the ratios and $30^{\circ}$ for the phase differences. Ducruix et al. have shown that the results

David R. Barraclough is in the Geomagnetism Unit, Institute of Geological Sciences, Edinburgh.

I/E $\quad t-\varepsilon$

Harwood \& Malin

$0.128^{\circ}$

Yukutake \& Cain

$0.32105^{\circ}$

Ducruix et al.

$0.1744^{\circ}$

of Yukutake and Cain are not compatible with simple induction models. Considering the uncertainties, the results of the other two studies are in general agreement.

Ducruix et al. have used their values of $I / E$ and $t-\varepsilon$ to test a series of four-layer models of mantle conductivity. The results indicate that the maximum value of the conductivity (which occurs at the bottom of the mantle) is approximately $100 \Omega^{-1} \mathrm{~m}^{-1}$. The results of Harwood and Malin, which are based on a much more extensive collection of data, are also consistent with a maximum value of $100 \Omega^{-1} \mathrm{~m}^{-1}$.

Runcorn (Trans. Am. geophys. Un. 36, $191 ; 1955)$ showed that, for a change of the core field with a duration of 4 years to be detectable at the Earth's surface, the conductivity of the lower region of the mantle cannot exceed $100 \Omega^{-1} \mathrm{~m}^{-1}$. Since the sudden change of secular acceleration about 1970 lasted for less than 4 years we are lead to the same upper limit for the conductivity as that given by the RV studies.

These two pieces of evidence thus cast considerable doubt on the higher estimates of lower mantle conductivity such as the figure of $10^{4} \Omega^{-1} \mathrm{~m}^{-1}$ proposed by Stacey $e t$ al. (Trans. Am. geophys. Un. 59, 1027; 1978 ) and that of $10^{5} \Omega^{-1} \mathrm{~m}^{-1}$ favoured by Alldredge (J. geophys. Res. 82, 5427; 1977).

Much work remains to be done in extending the studies of $\mathrm{RV}$ and the sudden changes in secular acceleration and in applying the results to determining more accurately the conductivity distribution in the lower mantle. The primary requirement is a good set of data, well distributed over the Earth's surface. The existing network of permanent magnetic observatories, which provide the most valuable data for such studies, has a very patchy distribution, very dense in Europe but very sparse throughout most of the Southern Hemisphere. Satellite surveys, such as that of the intensity and direction of the geomagnetic field currently being performed by Magsat, can only help if they are repeated at frequent intervals and even then they present their own set of problems. As far as the practical uses of the secular variation in the production of magnetic charts are concerned, we probably have sufficient knowledge of RV to attempt to correcl secular variation estimates for its effects. The problem with the sudden changes is that they are, at present, completely unpredictable. Here, frequently repeated satellite surveys might help in detecting such changes as soon as possible after their occurrence. 